\title{
Clinical, Radiographic and Histologic Evaluation of Regional Odontodysplasia: an Unusual Case Report with 6-Year Follow-Up
}

\author{
María Fe Riolobos González ${ }^{1, *}$, Lourdes García-Navas Fernández de la Puebla ${ }^{1}$, Fernando Costa Ferrer ${ }^{1}$, Alvaro Zubizarreta \\ Macho $^{2}$, Leticia Chico Hernández ${ }^{1}$, Carmen López Moreno ${ }^{1}$ and Anabella Reyes Ortiz ${ }^{1}$
}

${ }^{1}$ Dentist, Department of Pediatric Dentistry, Alfonso X El Sabio University, Madrid, Spain

${ }^{2}$ Department of Endodontics, Alfonso X El Sabio University, Madrid, Spain

*Corresponding author: María Fe Riolobos González, Professor, Department of Pediatric Dentistry, Alfonso X El Sabio University, Madrid, Spain, E-mail: mriolgon@uax.es

Received: 07 Oct, 2020 | Accepted: 21 Oct, 2020 | Published: 01 Feb, 2021

Citation: Riolobos González MF, García-Navas Fernández de la Puebla L, Costa Ferrer F, Zubizarreta Macho A, Chico Hernández L, et al. (2020) Clinical, Radiographic and Histologic Evaluation of Regional Odontodysplasia: an Unusual Case Report with 6-Year Follow-Up. Int J Dent Oral Health 7(2): dx.doi.org/10.16966/2378-7090.341

Copyright: (C2020 Riolobos González MF, et al. This is an open-access article distributed under the terms of the Creative Commons Attribution License, which permits unrestricted use, distribution, and reproduction in any medium, provided the original author and source are credited.

\begin{abstract}
Background: Regional odontodysplasia is a rare abnormality of unknown aetiology affecting dental tissues derived from the mesoderm and ectoderm. It occurs mostly in women, with no racial predilection. It is often located in the upper jaw and tends to affect a single quadrant. It can involve the temporary as well as the permanent dentition. The aetiology is unknown; however, local, systemic or genetic factors may be involved. The affected teeth are clinically hypoplastic and hypocalcified, radiographically demonstrating the appearance of "ghost teeth".

Case report: We present the case of a 9-year-old girl who came to the clinic due to an infection located in the first lower left molar that was half-buried gingivally. Previous medical history reflected a diagnosis of regional odontodysplasia at 2 years of age in the third quadrant of the temporal dentition. Clinically, she presented at birth with a slight facial haemangioma in the left hemi-mandibular region until 6-8 months. Intraorally, dental absences were observed in the third quadrant, except for the first left permanent molar, with hypoplastic features and a related abscess. Radiographically, the germs of the central and lateral incisors, canines and lower premolars demonstrated features of "ghost teeth". The first permanent left mandibular molar was removed, with subsequent prosthetic rehabilitation using a removable acrylic replacement plate.
\end{abstract}

Conclusions: The treatment plan for this anomaly should be determined by the patient's age, presenting symptoms, and aesthetic and/or functional needs. There is controversy over whether the affected teeth should be maintained for normal development of the jaws or should be extracted to avoid serious infections.

Keywords: Odontodysplasia; Ghost teeth; Odontogenic dysplasias; Tooth abnormalities

Abbreviations: RO: Regional Odontodysplasia; PAX9: Paired Box 9; CT: Computerized axial Tomography; CBCT: Conical Beam Computed Tomography; PRF: Platelet-Rich Fibrin

\section{Background}

Dental anomalies, depending on the stage of dental development in which they occur, maybe of any number, size, shape, structure and/or colour; thus, the classification of dental anomalies have been described in detail by Prescott G, et al. [1,2]. Regional odontodysplasia (RO) is defined as one of the "relatively rare" anomalies of dental development that affects dental tissues derived from the mesoderm and ectoderm, such as the enamel, dentine, pulp and follicle of both dentitions [3]. According to the International Statistical Classification of Diseases and Related Health Problems (tenth revised version of 2007), regional odontodysplasia is classified as K00.4 [4].

It was initially defined by Hitchin in 1934 [5]. The first case described in the literature was published by McCall JO, et al. [6].
However, the term "odontodysplasia" was coined by Zegarelli EV, et al. [7]; later on, Pindborg added the prefix "regional" due to its tendency to affect a single quadrant [8].

To date, many terms have been used to define this pathology, such as "ghost teeth"; "imperfect odontogenesis"; "unilateral dental malformation"; "localized hypoplasia"; "imperfect amelogenesis"; or "odontogenic dysplasia”.

Epidemiologically, RO has no predilection for any race or ethnicity, although, regarding sex, some differences have been observed, as RO is more frequent in women than in men (1.7:1). This pathology is predominant in the upper jaw (55.9\%) compared to the lower jaw (33.5\%) [9]. RO is normally unilateral and rarely crosses the midline, with the central, lateral and canine incisors being the most affected 
teeth, although the rest of the teeth in the arch may be involved. Bilateral maxillary involvement has been described in $4.3 \%$ and mandibular involvement in $9.3 \%$ of cases [10].

RO has a prevalence of less than 1:1.000.000, and there have been approximately 168 cases published in the English language up to 2019 [3].

Clinically, RO affects both the temporary and permanent dentition; when the primary teeth are involved, permanent successors are often affected as well. However, affected permanent teeth can replace healthy temporary teeth, and rarely, a healthy permanent tooth can replace an affected deciduous tooth. The degree of dental involvement can vary even in the same quadrant. $\mathrm{RO}$ is usually observed in consecutive teeth, without healthy intermediate teeth. The first permanent molars are only affected if the adjacent primary molar shows RO [10].

Although the aetiology of RO is unknown, several hypotheses have been suggested. RO is not a hereditary condition but seems to be related to local factors during dental development. One theory suggests that it can be produced by a somatic mutation of the PAX9 gene occurring at an early stage of development, which may predispose the outgrowth of an anomaly such as regional odontodysplasia [11-13].

Another supposed aetiological factor involves the presence of an alteration in the local circulation, giving rise to ischaemia in the area and thus affecting odontogenesis. In addition, this assumption is supported by the fact that some cases of OR are associated with haemangiomas in areas adjacent to the affected teeth [14].

Other factors causing OR have been mentioned, such as neural crest cell defects, viral infections, local trauma, Rh incompatibility, hyperpyrexia, ionizing radiation, teratogenic pharmacological agents and nutritional and/or metabolic alterations. OR has been associated with the presence of nevus, hypophosphatasia, hypocalcaemia, ectodermal dysplasia, and hydrocephalus; however, most cases of OR are not associated with medical alterations [9,14-17].

Despite the unknown aetiology, regional odontodysplasia demonstrates clinical, radiographic and histological pathognomonic signs on which its diagnosis is based. Histologically, all tooth tissues are affected. The structure of the enamel is hypoplastic and hypocalcified, and the enamel has a variable thickness and an uneven surface [18]. Dentin is observed in histological slices with an interruption of the dentinal tubules due to the presence of interglobular dentin. The presence of this interglobular dentin together with amorphous basophilic areas of non-collagenous material has been suggested to be a pathognomonic feature of RO [14].

The pulp chamber is large in $\mathrm{RO}$, with various degrees of calcification. In terms of the cement, some authors claim that it is only slightly affected in RO. Others claim that the cement may be thin, may have a globular structure, and may even be absent [14].

Clinically, RO cases demonstrate abnormal morphology and yellow-brown coloration. They exhibit an irregular porous surface with extreme erosion. They are also susceptible to fracture. Other common clinical signs after the eruption of RO teeth are gingival enlargement, periapical infections, and abscess formation [17].

Radiographically, affected developing teeth display a dental follicle and an expanded pulp chamber as well as short roots with open apical foramen. There is a lack of contrast between the enamel and dentine, and both are less radiopaque, resulting in the appearance of "ghost teeth". The eruption is delayed relative to the contralateral side, with even a mandibular radio density altered by a decrease in trabeculae.
Occasionally, unerupted teeth are surrounded by pericoronal radiolucent regions with clear boundaries, which resemble a cyst or tumour $[15,18]$.

The aim of this work is to report a case of regional odontodysplasia after 6 years of follow-up, as well as to review what has been published in the literature in terms of this pathology, in order to achieve correct diagnosis and early treatment.

\section{Case Report}

The case of a 9-year-old female paediatric patient referred to the Paediatric Dentistry Service of the Alfonso X El Sabio University (Madrid, Spain) for pain and an abscess located in the left posterior mandibular region is presented. Upon anamnesis, the mother referenced a traumatic delivery with forceps. At the time of birth, she presented with a purple facial spot on the left mandible, which represented possible paediatric haemangioma and was present until 6-8 months of age. Systemic medical or dental diseases were not referenced by any member of her family. At the age of 2 , she was diagnosed with RO in the third quadrant at a hospital where all temporary teeth in the third quadrant were extracted due to fistulas, cavities and submerged teeth. She was treated with a removable resin plate for the replacement of teeth 71-72-73-74-75 but did not adapt to it. She continued to receive dental check-ups until she came to our service due to pain in the first permanent left mandibular molar (Figure 1).

Upon intraoral exploration, a vestibular abscess was observed in the $3^{\text {rd }}$ quadrant, with a hypoplastic appearance of the first left permanent molar. In the $2^{\text {nd }}$ quadrant, molar extrusion was observed due to the lack of antagonist pieces. The alveolar ridge was heavily reabsorbed. The rest of the quadrants had a normal appearance and displayed normal tooth replacement according to age (Figure 2). Upon radiographic examination, the germs of the central, lateral, canine, premolars and first molar of the $3^{\text {rd }}$ quadrant demonstrated more initial

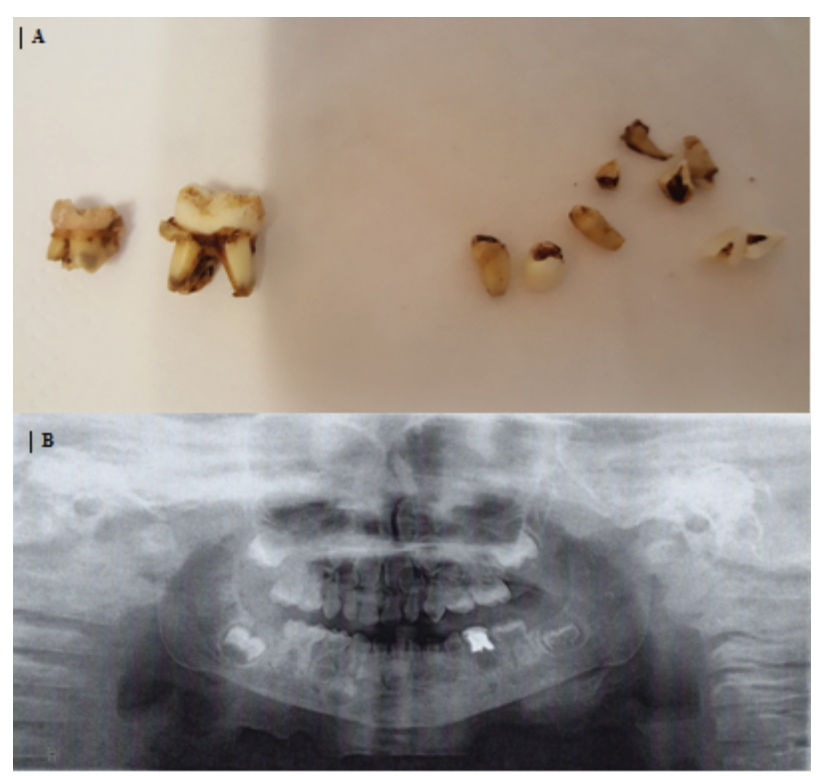

Figure 1: (A) Temporary teeth extracted from the $3^{\text {rd }}$ quadrant at the age of 3 years old.

(B) Panoramic X-ray at the age of 3 years when regional odontodysplasia was diagnosed. 


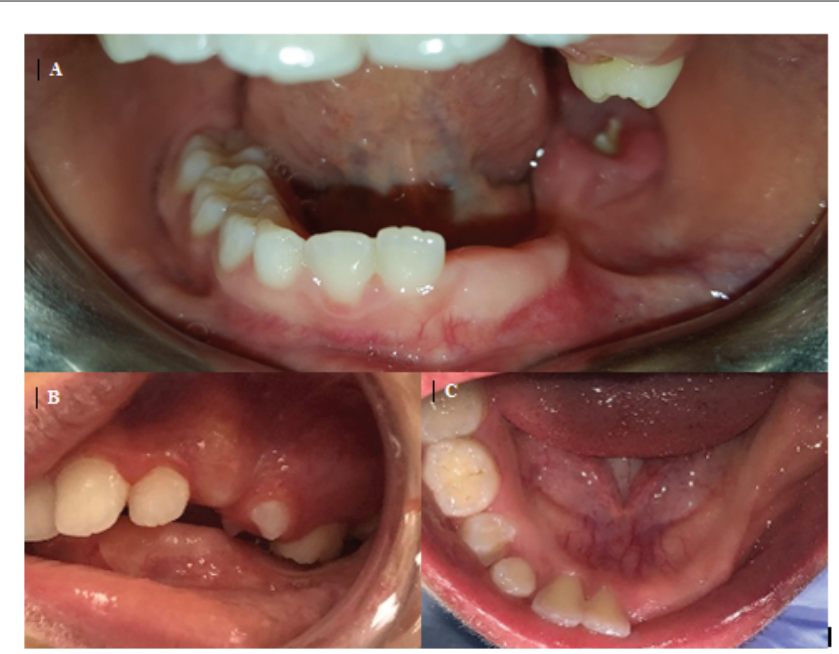

Figure 2: (A) Hypoplastic eruption of the mandibular left first molar with gingival overgrowth and a second extrusion of the upper left temporal molar.

(B) Intraoral image of the lower jaw. Decreased left alveolar ridge.

(C) Left lateral intraoral image. Extrusion of upper molars with a lack of prosthetic space.

stages of development in addition to a delay in eruption compared to the contralateral teeth. Ghost tooth features were observed due to the poor demarcation between the enamel and dentin and the large pulp chambers. However, the second permanent molar had developed similar to its contralateral counterpart (Figures 3,4).

The initial emergency treatment in the hospital consisted of extraction of the first left mandibular permanent molar with biopsy of the apical lesion of the molar after extraction. Computerized axial tomography $(\mathrm{CT})$ was performed to assess the proximity of the lower dental canal to the first left lower permanent molar (Figures 5,6).

The results of the histological test demonstrated the cystic formation of a collagen tissue wall with abundant inflammatory chronic lymphoplasmacytic interstitial infiltrates, and cholesterol granulomas and associated dystrophic microcalcification foci were observed; the epithelial lining was observed to be of the polystyrene pavement type without keratin formation. A diagnosis of an odontogenic cyst, compatible with a root cyst, was made. Subsequently, the cyst was prosthetically rehabilitated with a removable aesthetic acrylic plate for repositioning of the left central incisor as well as the left lateral and canine mandibular incisors with acrylic extension during occlusion to stimulate dental eruption and avoid extrusion of the antagonist's teeth (Figures 7,8 ). On panoramic radiography carried out during the year of revision, the germs of the distalised ghost teeth were observed occupying the space of the first extracted permanent molar (Figure 9).

The long-term treatment provides orthodontic treatment for the intrusion of second quadrant teeth when apical closure occurs, with adjustment of the occlusal plane. The exodontia of the affected teeth will be assessed if they do not achieve normal dental development and/ or if they demonstrate a poor prognosis, with subsequent rehabilitation of the affected sector through bone regeneration, if necessary, and implant-supported prostheses.

\section{Discussion and Conclusions}

The case in question is that of a 9-year-old girl affected by left

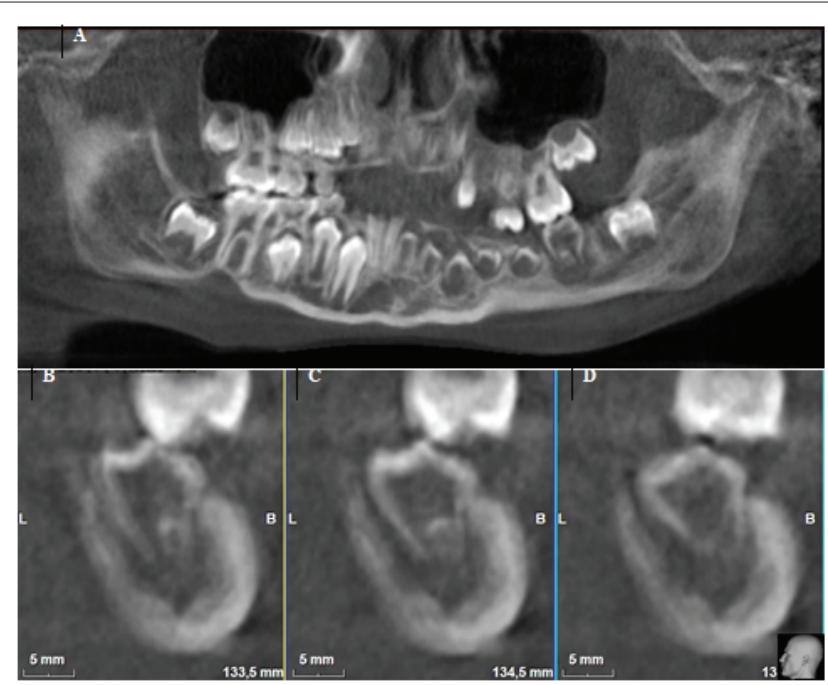

Figure 3: (A) Panoramic radiography at 8 years of age, in which "ghost teeth" are observed in the third quadrant as well as extrusion of the antagonist teeth.

(B-C-D) Frontal section of CT, where the difference in the two lower permanent molars is observed with abnormal development of the second left mandibular premolar.

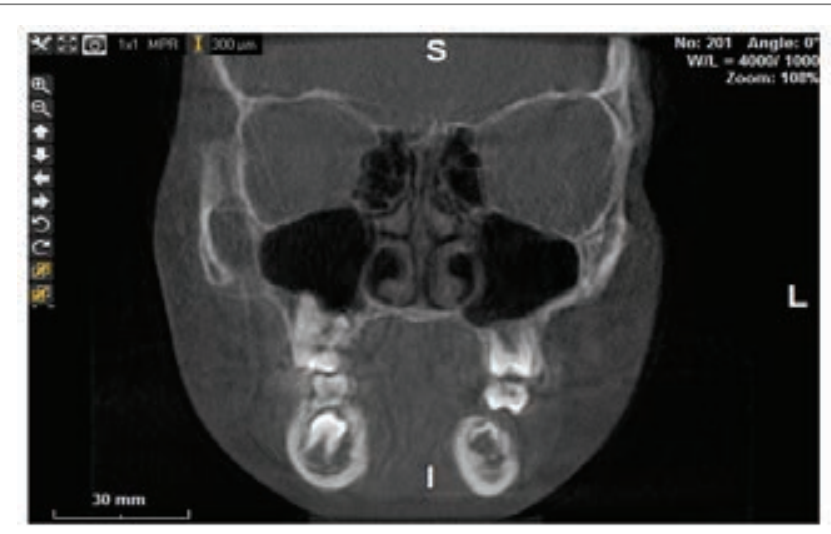

Figure 4: Coronal section at the level of the first mandibular premolar where the dental anomaly of the third quadrant is observed.

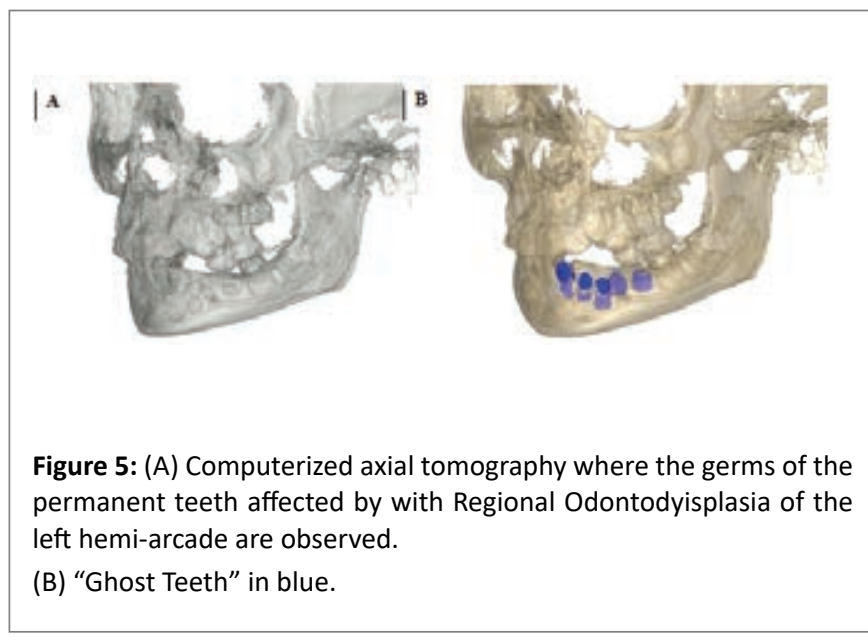




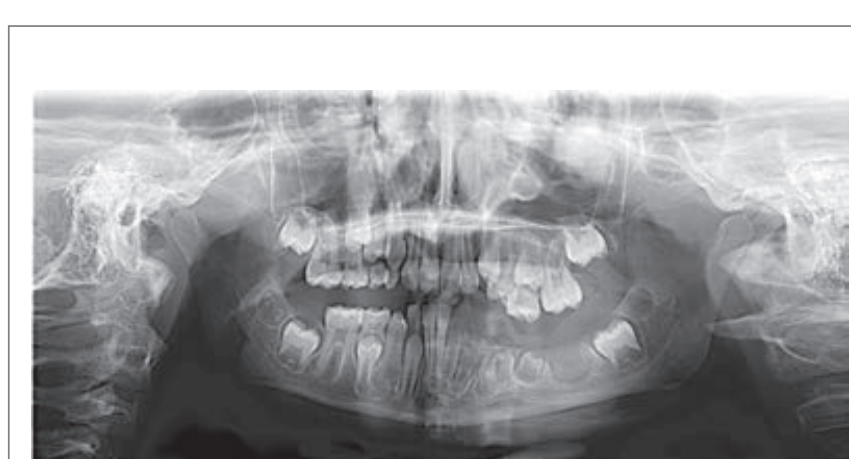

Figure 6: Panoramic radiography showing the teeth with a "ghost teeth"; appearance; and absence of tooth 36 after extraction. The molars of the second quadrant have beenwere extruded (January 2019).

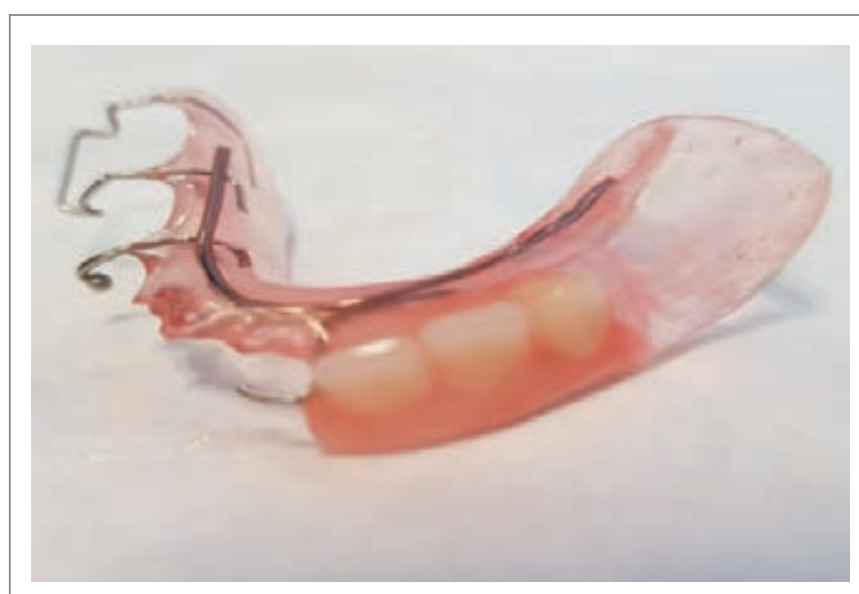

Figure 7: Removable resin plate for the replacement of teeth 31,32 and 33 .

mandibular regional odontodysplasia in both the temporary and permanent dentition without exceeding the midline. This unusual location accounts for $13 \%$ of published cases [10].

Alotaibi $\mathrm{O}$, et al. Analysed cases published in the literature from 1953 to 2017 in the English language to obtain clinical and epidemiological data, following the protocols of the PRISMA guide. They found 161 cases of patients between the ages of 1 and 25 years when reported, with a female predilection (1.37:1). The upper jaw was the most affected region (55.9\%) with respect to the mandible (33.5\%). If there was only one quadrant affected, the order of affected regions was as follows: left jaw, right jaw, left jaw and right jaw. They found that RO affected the two dentitions, the temporary and permanent dentition, in 75 cases; only the temporary dentition was affected in $19.35 \%$ of cases, and only the permanent dentition was affected in $34.2 \%$ of cases. In the temporary dentition, all teeth were affected equally; however, in the permanent dentition, the anterior sector was the most affected. The authors reported rash failures as the most frequent signs and symptoms, followed by cortical widening due to abscess formation [10].

Multiple aetiologies have been proposed, with the defective vascular function being the most accepted. The association of vascular nevi in the skin covering the areas where RO occurs suggests that a

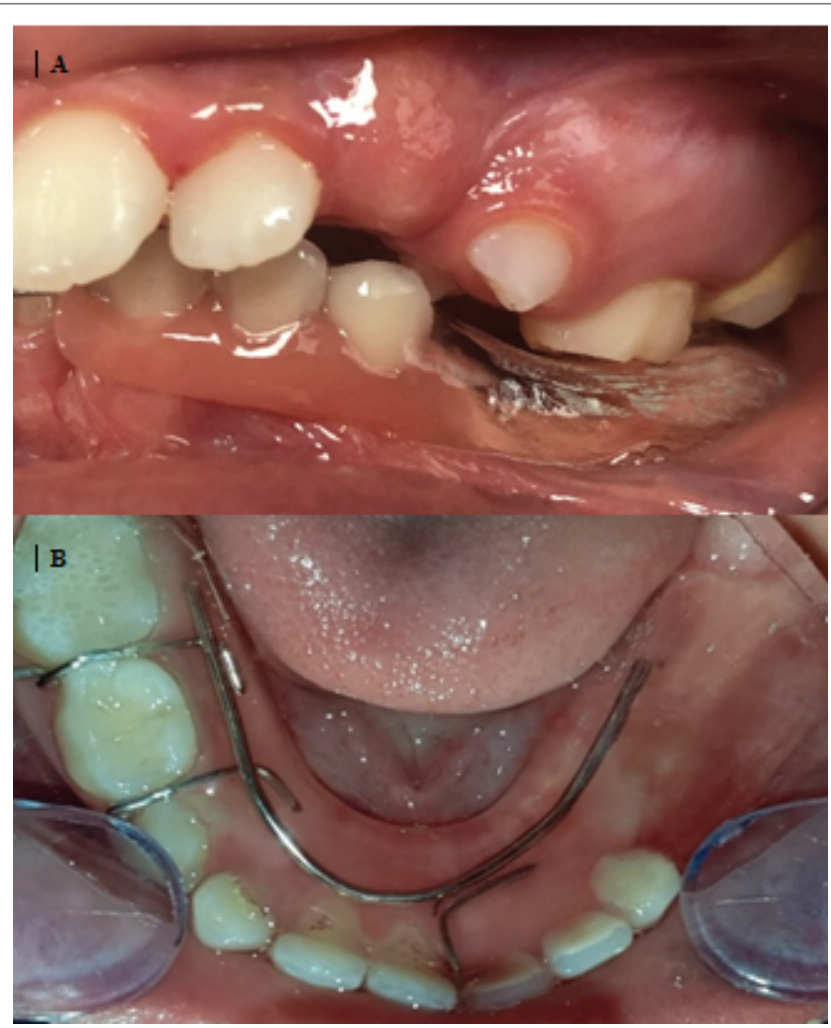

Figure 8: (A) Intraoral photograph of thewith removable resin plate within occlusion.

(B) Intraoral photograph of the lower arch with a removable resin plate in the mouth.

local vascular defect may be involved in the pathogenesis of RO. These nevi are pale pink and gradually disappear with age [19]. In the case presented here, the patient had a haemangioma at birth that remained for up to 8 months after birth at the left mandibular level. The literature suggests that there may be a common aetiology for dental agenesis and RO, but more studies are needed to determine the role of the PAX9 gene in these processes. Genetic testing of patients and exome/genome analysis is required to obtain more information on the aetiology of this rare abnormality. Other conditions that share some characteristics with regional odontodysplasia include dentin dysplasia type I and II, rickets, hypophosphatasia, amelogenesis imperfecta, and dentinogenesis imperfecta [11-13].

Regarding the treatment of regional odontodysplasia, it is useful to perform a three-dimensional evaluation using axial computed tomography (CT) or conical beam computed tomography (CBCT). These techniques allow us to obtain the morphology of the affected teeth, the volume of the pulp chamber, the density of the dental tissues, the exact location of $\mathrm{RO}$ and its relationship with adjacent structures.

The reason for the reported case was the presence of pain at the level of the first left permanent mandibular molar. It was hypoplastic in appearance, with a related abscess. The prognosis of the affected teeth was poor since they presented with alterations of all their tissues as well as eruption; in addition, the roots were very short, and the apexes were opened. Early diagnosis of this pathology is considered an important critical factor in treatment, although there is controversy in the literature regarding the maintenance or extraction of affected 


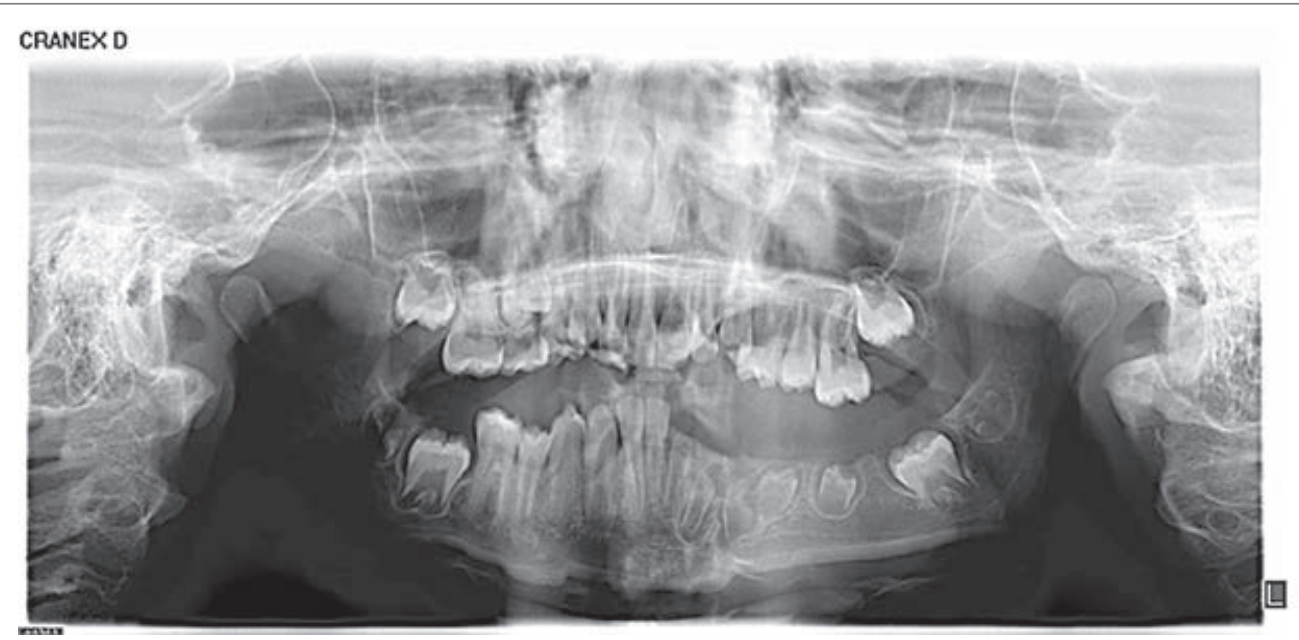

Figure 9: Panoramic radiography showing lack of corono-radicular development and deviation of the trajectory of the left lower premolars occupying the space of the extracted first left permanent molar extracted.

teeth. Thus, some authors advocate conservative treatment to avoid a substantial reduction in marginal ridge height. For this, they apply dietary measures, oral hygiene, fluoride application, temporary restoration, and pre-formed metal crown placement, among other alternatives. Other authors believe that treatment should be more radical to prevent serious future infections $[9,14,20-23]$.

Premature exodontia of the temporary teeth can have undesirable consequences, such as a delay in the eruption of the successor permanent teeth as well as an alteration in the development of the alveolar bone in this area. The published literature indicates that if the affected teeth present with abscesses, they should be extracted, and the toothless areas should be restored with removable acrylic appliances to maintain aesthetic and masticatory functions, to avoid the dental extrusion of the antagonist's teeth to the affected ones, to achieve the preservation of the space and the normal vertical dimension, and to diminish the psychological effects of the premature loss of teeth. However, patients often have progressive bone resorption, atrophied ridges, and reduced vertical dimensions in the affected area, which later makes it difficult to insert future dental implants when skeletal growth is complete. Autografts, allografts and artificial bone substitutes have been used for this purpose but with limitations [9].

Regeneration of reabsorbed alveolar bone may be beneficial for patients suffering from early tooth loss with subsequent bone resorption to facilitate the insertion of dental implants. Platelet-rich fibrin (PRF) has been shown to be useful for soft tissue treatment and bone regeneration [24].

With regard to orthodontic treatment, caution must be taken since the roots of these teeth are short or non-existent, and there is a low density of the bone around the roots. Only slight forces should be applied to this situation.

In this case, multidisciplinary treatment was required (paediatric dentist, maxillofacial surgeon, orthodontist) to achieve correct oral health, preserving the masticatory function, the control of bone development and general dentistry, the monitoring of tooth development with $\mathrm{RO}$ for maintenance or surgical treatment if necessary as well as subsequent prosthetic rehabilitation. From 2017 to November 2019, 7 more articles reporting 7 clinical cases were published and provided similar data to those collected by Alotaibi O, et al. $[9,13,17,19,24-28]$.

Thus, in patients with regional odontodysplasia the type of rehabilitation in each case will depend on the number of affected teeth, the integrity of the teeth, the age of the patient, previous experiences and aesthetic and functional needs, although the most viable option would be a removable prosthesis since there are no studies that have determined the success of the placement of an implant-supported prosthesis in young patients.

In conclusion, regional odontodysplasia is a rare disease with severe dental involvement. After 168 cases published in the English language in 66 years, the aetiology is currently unclear. Three-dimensional imaging and CT data can greatly improve the diagnosis and treatment of RO.

\section{References}

1. Martin J, Sanchez B, Tarilonte ML, Castellanos L, Llamas JM, et al. (2012) Inherited dental abnormalities and dysplasias. Av Odontoestomatol 28: 287-301.

2. Prescott G (1976) Oral Facial Genetics. Saint Louis, Mosby.

3. Crawford PJ, Alfred MJ (1989) Regional odontodysplasia: A bibliography. J Oral Pathol Med 18: 251-263.

4. International Statistical Classification of Diseases and Related Health Problems (1995) $10^{\text {th }}$ Revision. Washington DC.

5. Hitchin AD (1934) Unerupted deciduous teeth in a youth aged 15 1/2. Br Dent J 56: 631-633.

6. McCall JO, Wold SS (1952) Clinical Dental Roentgenology. 3rd edition, WB Saunders, Philadelphia.

7. Zegarelli EV, Kutscher AH, Applebaum E, Archard HO (1963) Odontodysplasia. Oral Surg 16: 187-193.

8. Pindborg JJ (1970) Pathology of the dental hard tissues. $1^{\text {st }}$ edition, WB Saunders, Philadelphia.

9. Koruyucu M, Yaman D, Seymen F, Demirel K, Gencay K (2019) Management of regional odontodysplasia: a 10-year-follow-up case report and literature review. Eur Oral Res 1: 111-116. 
10. Alotaibi O, Alotaibi G, Alfawaz N (2019) Regional odontodysplasia: An analysis of 161 cases from 1953 to 2017. Saudi Dent J 31: 306-310.

11. Ceballos DM, Espinal GE, Jones M (2015) Development Abnormalities and Dental Training: Odontodysplasia. Int J Odontostomatol 9: 129-136.

12. Mostowska A, Biedziak B, Trzeciak WH (2006) A novel mutation in PAX9 causes familial form of molar oligodontia. Eur J Hum Genet 14: 173-179.

13. Koskinen $\mathrm{S}$, Keski-Filppula $\mathrm{R}$, Alapulli $\mathrm{H}$, Nieminen $\mathrm{P}$, Anttonen $\mathrm{V}$ (2019) Familial oligodontia and regional odontodysplasia associated with a PAX9 initiation codon mutation. Clin Oral Investig 23: 41074111.

14. Orta PB, Villar BB, Martinez MRM, Ferrer FC (2004) Odontodisplasia regional: a propósito de un caso. Odontol Pediatr (Madrid) 12: 51-55.

15. Hamdan MA, Sawair FA, Rajab RD, Hamdan AM, Al-omari IK (2004) Regional odontodysplasia: a review of the literature and report of a case. Int J Paediatr Dent 14: 363-370.

16. Al-Mullahi AM, Toumba KJ (2016) Regional Odontodysplasia with Generalised Enamel Defect. Case Rep Dent 3: 457-467.

17. Bowden E, Carroll C, Gill M, Llewelyn R (2018) Cervicofacial infection in a 3-year-old child with regional odontodysplasia: A case report and literature review. J Oral Maxillofac Pathol 22: S117-S120.

18. Gardner DG (1974) The dentinal changes in regional odontodysplasia. Oral Surg Oral Med Oral Pathol 38: 887-897.

19. De Sá Cavalcante D, Sr Fonteles C, Ribeiro TR, Kurita LM, Pimenta AVM, et al. (2018) Mandibular Regional Odontodysplasia in an 8-year-old Boy showing Teeth Disorders, Gubernaculum Tracts, and Altered Bone Fractal Pattern. Int J Clin Pediatr Dent 11: 128-134.
20. Jahanimoghadam F (2016) Dental Anomalies: An Update. Adv Hum Biol 6: 112-118.

21. Saberi E, Ebrahimipour S (2016) Evaluation of developmental dental anomalies in digital panoramic radiographs in Southeast Iranian Population. J Int Soc Prev Community Dent 6: 291-295.

22. Matsuyama J, Tanaka R, lizawa F, Sano T, Kinoshita-Kawano S, et al. (2014) Clinical and Radiographic Findings and Usefulness of Computed Tomographic Assessment in Two Children with Regional Odontodysplasia. Case Rep Dent 1-5.

23. Cahuana A, González Y, Palma C (2005) Clinical management of regional odontodysplasia. Pediatr Dent 27: 34-39.

24. Abdel-Kader MA, Abdelazeem AF, Ahmed NEB, Khalil YM, Mostafa MI (2019) Oral rehabilitation of a case with regional odontodysplasia using a regenerative approach-A case report and a review of literature. Spec Care Dent 39: 330-339.

25. Cunha JLS, Santana AVB, da Mota Santana LA, Santos DM, de Souza Amorim K, et al. (2019) Regional Odontodysplasia Affecting the Maxilla. Head Neck Pathol 14: 224-229.

26. Rashidian A, Afsharian A, Azarshab M, Zarrabian T (2013) Regional Odontodysplasia: Report of a case. J Dent (Shiraz) 14: 197-200.

27. Masis $P$, Montero O (2009) Regional odontodysplasia: Case report in Costa Rica and literature review. Rec Cient Odontol 5: 34-38.

28. Hegazi F, Hassan M (2018) Regional odontodysplasia crossing the midline. J Dent Child (Chic) 85: 88-91. 